\title{
Association between body mass index and presence of carotid plaque among low-income adults aged 45 years and older: a population-based cross-sectional study in rural China
}

\author{
Yongzhong Lou ${ }^{1}$, Bin $\mathrm{Li}^{1}$, Lan Su${ }^{1}$, Zhenhong Mu${ }^{1}$, Minghao Sun ${ }^{1}$, Hongfei Gu ${ }^{1}$, \\ Jingxian $\mathbf{N i}^{2,3,4}$, Yanan Wu $\mathbf{u}^{2,3,4}$, Jun Tu $\mathbf{u}^{2,3,4}$, Jinghua Wang ${ }^{3,4,5}$ and Xianjia $\mathbf{N i n g}^{3,4,5}$ \\ ${ }^{1}$ Department of Neurology, Tianjin Haibin People's Hospital, Tianjin, China \\ 2 Department of Neurology, Tianjin Medical University General Hospital, Tianjin, China \\ ${ }^{3}$ Department of Epidemiology, Tianjin Neurological Institute, Tianjin, China \\ ${ }^{4}$ Tianjin Neurological Institute, Key Laboratory of Post-Neuroinjury Neuro-Repair and Regeneration in Central Nervous \\ System, Ministry of Education and Tianjin City, Tianjin, China \\ ${ }^{5}$ Center of Clinical Epidemiology, Tianjin Medical University General Hospital, Tianjin, China \\ Correspondence to: Bin Li, email: libin_1971_2008@sina.com \\ Xianjia Ning, email: xjn0906@gmail.com \\ Keywords: carotid plaque, body mass index, ultrasonography, low-income population, epidemiology \\ Received: February 08, $2017 \quad$ Accepted: April 17, $2017 \quad$ Published: May 04, 2017
}

Copyright: Lou et al. This is an open-access article distributed under the terms of the Creative Commons Attribution License 3.0 (CC BY 3.0), which permits unrestricted use, distribution, and reproduction in any medium, provided the original author and source are credited.

\section{ABSTRACT}

Carotid plaque is a good surrogate endpoint for assessing arterial atherosclerosis, and atherosclerosis is a reliable predictor of cardiovascular diseases. However, the effect of body mass index on carotid plaque is unknown. Therefore, we aimed to explore the association between body mass index and carotid plaque in a low-income Chinese population. Residents aged $\geq \mathbf{4 5}$ years and free of stroke and cardiovascular diseases were enrolled and divided into four groups based on body mass index. B-mode ultrasonography was performed to measure carotid plaque. The mean age of participants was $\mathbf{5 9 . 9 2}$ years overall. Significant correlations were observed between the presence of carotid plaque and male sex, older age, systolic blood pressure, fasting plasma glucose, and low-density lipoprotein cholesterol among the different BMI subgroups. Male sex increased the risk of carotid plaque in the overweight and obese groups. Older age and high level of low-density lipoprotein cholesterol were the independent risk factor for carotid plaque in four groups. Increased systolic blood pressure was an independent risk factor in the normal-weight, overweight, and obese groups; however, fasting plasma glucose was only significant in the normal-weight group. Thus, controlling the levels of low-density lipoprotein cholesterol, systolic blood pressure, and fasting plasma glucose is required to reduce carotid plaque risk.

\section{INTRODUCTION}

The World Health Organization stated that 17.5 million people died of cardiovascular diseases (CVDs) in 2012, comprising $31 \%$ of all deaths globally. An estimated 7.4 million of those were due to coronary heart disease, and 6.7 million were due to stroke [1]. Of the 16 million deaths of individuals $<70$ years of age due to non-communicable diseases, $82 \%$ occurred in low- and middle-income countries, and $37 \%$ were caused by CVDs
[1]. CVDs are the leading cause of death both in Western countries and in China, accounting for $40 \%$ of deaths in China in 2012 [2]. Among those, 44.6\% of deaths in rural areas and $42.5 \%$ in urban areas were CVD-related [2]. Stroke is the second most common cause of death; Feigin et al. reported that the age-standardized stroke rate in low- and middle-income countries is 1.24-fold higher than that in high-income countries [3]. Furthermore, the stroke prevalence among patients $\leq 75$ years in low- and middle-income countries is three times higher than that in 
high-income countries [3]. Thus, individuals with CVD, or who are at high risk for it, require early detection and management to prevent or delay end events.

Several studies have shown that atherosclerosis is a reliable predictor for CVDs and that that presence of carotid plaque is a better surrogate endpoint for assessing arterial atherosclerosis lesions than is carotid intima-media thickness [4-9]. Several studies have also investigated risk factors for carotid plaque, such as age, sex, cigarette smoking, alcohol consumption, body mass index (BMI), education levels, and so on [6, 9-20]. However, there has been no consensus on the effect of BMI on carotid plaque. A study from Iceland showed that BMI protected against carotid plaque. Sturlaugsdottir et al. [9] demonstrated that after adjustment for other confounding factors, the risk of developing carotid plaque decreased by $28 \%$ for each 5-unit increase in BMI (odds ratio [OR], 0.72; 95\% confidence interval [CI], 0.64-0.82; $P<0.001)$. In contrast to those results, West et al. [15] reported that BMI appeared to be a risk factor for carotid plaque among 2,448 young Finnish adults. They studied the risk of carotid atherosclerotic plaque in their adulthood following exposure to parental smoking in childhood and found that the average BMI in individuals without carotid plaque was $17.8 \mathrm{~kg} / \mathrm{m}^{2}$, while that in individuals with carotid plaque was $19.1 \mathrm{~kg} / \mathrm{m}^{2}$. Furthermore, several studies have demonstrated that BMI had no association with carotid plaque $[8,19,21]$. There are even fewer studies in Chinese populations. Because of the genetic differences between Chinese and Caucasian populations, conclusions from Caucasian populations regarding the associations between BMI and carotid plaque cannot be generalized to Chinese populations. Thus, the purpose of the present populationbased study was to determine the association between BMI and carotid plaque among a low-income population in rural China.

\section{RESULTS}

\section{Demographic characteristics for subjects}

In the present study, 4,012 participants were interviewed from among 5,380 eligible residents aged $\geq 45$ years during the study period. The response rate was $74.5 \%$. Finally, 3,789 subjects were included in our study, after excluding 223 residents with a previous history of CVD or stroke (Figure 1).

Among the 3,789 subjects, there were 1,560 men $(41.2 \%)$ and 2,229 women $(58.8 \%)$. The average age was $59.92(9.70)$ years overall, $61.13(9.90)$ years for men, and $59.07(9.47)$ years for women $(P<0.001)$. The mean length of formal education was 5.48 years, and $17.4 \%$ of participants had never received a formal education (8.8\% of men and $23.4 \%$ of women); $37.9 \%$ had gained a formal education $\geq 6$ years. Men were more likely to have higher educational attainment, to be cigarette smokers, to consume alcohol, and to have hypertension; however, women had higher rates of illiteracy and obesity. Systolic blood pressure (SBP) and diastolic blood pressure (DBP) were higher in this population, with mean values of $146.42 \mathrm{mmHg}$ and $86.81 \mathrm{mmHg}$, respectively. Moreover, SBP and DBP were higher in men than in women, but BMI, total cholesterol (TC), triglycerides (TG), highdensity lipoprotein cholesterol (HDL-C), and low-density lipoprotein cholesterol (LDL-C) were greater in women than in men (Table 1).

\section{Prevalence of carotid plaque by demographic characteristics and risk factors}

Table 2 shows the prevalence of carotid plaque by demographic characteristics and risk factors for all participants in this study. There was a significantly higher prevalence of carotid plaque associated with male sex, older age, cigarette smoking, hypertension, and diabetes mellitus (DM) (all $P<0.001)$; the plaque prevalence descreased with increasing BMI $(P=0.008)$; meanwhile the prevalence of plaque decreased with advancing education level $(P<0.001)$.

\section{Differences in risk factors based on the presence or absence of carotid plaque}

There was a significant discrepancy in mean BMI, SBP, DBP, fasting plasma glucose (FPG), TC, LDL-C, and the LDL-C/HDL-C ratio between individuals with or without carotid plaque (all $P<0.05$ ). However, TG, HDL-C, and waist circumference were similar between individuals with or without carotid plaque (all $P>0.05$ ) (Table 3).

\section{The prevalence of carotid plaque in the stratified analysis}

Tables 4 and 5 show the prevalence of carotid plaque by BMI for all participants in this study. The prevalence of carotid plaque was higher for men and older individuals among all four weight groups (underweight, normalweight, overweight, and obese; all $P<0.05$ ). Carotid plaque prevalence decreased with advancing education level for all groups except the underweight group $(P=$ 0.090). Moreover, the prevalence of carotid plaque was higher in patients with hypertension and DM and among cigarette smokers in the normal-weight, overweight, and obese groups (all $P<0.05$ ).

Waist circumference, TC, and FPG were significantly higher in patients with carotid plaque in the normal-weight and overweight groups (all $P<0.01$ ). SBP 
Table 1: The demographical characteristic and risk factors in this population by gender.

\begin{tabular}{|c|c|c|c|c|}
\hline Risk factors & Total & Men & Women & $P$ \\
\hline Total: & $3789(100)$ & $1560(41.2)$ & $2229(58.8)$ & \\
\hline Age, means (SD), years & $59.92(9.70)$ & $61.13(9.90)$ & $59.07(9.47)$ & $<0.001$ \\
\hline Age group, n (\%) & & & & $<0.001$ \\
\hline $45 \sim 54$ years & $1236(32.6)$ & $430(27.6)$ & $806(35.2)$ & \\
\hline $55 \sim 64$ years & $1514(40.0)$ & $632(40.5)$ & $882(39.6)$ & \\
\hline $65 \sim 74$ years & $724(19.1)$ & $338(21.7)$ & $386(17.3)$ & \\
\hline$\geq 75$ years & $315(8.3)$ & $160(10.3)$ & $155(7.0)$ & \\
\hline Education, means (SD), years & $5.48(6.54)$ & $6.40(3.22)$ & $4.84(3.61)$ & $<0.001$ \\
\hline Education, $\mathrm{n}(\%)$ & & & & $<0.001$ \\
\hline 0 years & $659(17.4)$ & $137(8.8)$ & $522(23.4)$ & \\
\hline $1 \sim 6$ years & $1694(44.7)$ & $699(44.8)$ & $995(44.6)$ & \\
\hline$>6$ years & $1436(37.9)$ & $724(46.4)$ & $712(31.9)$ & \\
\hline Smoking status, $\mathrm{n}(\%)$ & & & & $<0.001$ \\
\hline Never smoking & $2840(75.0)$ & $664(42.6)$ & $2176(97.6)$ & \\
\hline Smoking & $949(25.0)$ & $896(57.4)$ & $53(2.4)$ & \\
\hline Alcohol consumption, $\mathrm{n}(\%)$ & & & & $<0.001$ \\
\hline Never drinking & $3198(84.4)$ & $999(64.0)$ & $2199(98.7)$ & \\
\hline Drinking & $591(15.6)$ & $561(36.0)$ & $30(1.3)$ & \\
\hline BMI & & & & $<0.001$ \\
\hline Underweight & $66(1.7)$ & $27(1.7)$ & $39(1.7)$ & \\
\hline Normal & $1232(32.5)$ & $557(35.7)$ & $675(30.3)$ & \\
\hline Overweight & $1603(42.3)$ & $653(41.9)$ & $950(42.6)$ & \\
\hline Obesity & $888(23.4)$ & $323(20.7)$ & $565(25.3)$ & \\
\hline Hypertension, n (\%) & $2583(68.2)$ & $1111(71.2)$ & $1472(66.0)$ & 0.001 \\
\hline Diabetes, $\mathrm{n}(\%)$ & $533(14.1)$ & $216(14.1)$ & $317(14.5)$ & 0.719 \\
\hline Central obesity, n (\%) & $2317(61.3)$ & $778(50.0)$ & $1539(69.2)$ & 0.001 \\
\hline $\mathrm{SBP}$, means $(\mathrm{SD}), \mathrm{mmHg}$ & $146.42(22.17)$ & $147.76(21.41)$ & $145.49(22.64)$ & 0.002 \\
\hline DBP, means (SD), mmHg & $86.81(11.40)$ & $88.50(11.22)$ & $85.62(11.39)$ & $<0.001$ \\
\hline BMI, means (SD), $\mathrm{Kg} / \mathrm{m}^{2}$ & $25.57(3.68)$ & $25.20(3.44)$ & $25.82(3.82)$ & $<0.001$ \\
\hline FPG, means (SD), mmol/L & $5.92(1.57)$ & $5.91(1.42)$ & $5.93(1.67)$ & 0.660 \\
\hline $\mathrm{TC}$, means $(\mathrm{SD}), \mathrm{mmol} / \mathrm{L}$ & $4.87(1.09)$ & $4.62(1.00)$ & $5.04(1.11)$ & $<0.001$ \\
\hline $\mathrm{TG}$, means $(\mathrm{SD}), \mathrm{mmol} / \mathrm{L}$ & $1.76(1.24)$ & $1.61(1.24)$ & $1.87(1.22)$ & $<0.001$ \\
\hline HDL-C, means (SD), mmol/L & $1.46(0.46)$ & $1.39(0.43)$ & $1.50(0.48)$ & $<0.001$ \\
\hline LDL-C, means (SD), mmol/L & $2.70(1.25)$ & $2.61(1.20)$ & $2.76(1.28)$ & $<0.001$ \\
\hline LDL-C/HDL-C ratio & $2.02(1.22)$ & $2.05(1.32)$ & $1.99(1.14)$ & 0.103 \\
\hline
\end{tabular}

SD, standard deviation; SBP, systolic blood pressure; DBP, diastolic blood pressure; BMI, body mass index; FPG, fasting plasma glucose; TC, total cholesterol; TG, triglycerides; HDL-C, high density lipoprotein cholesterol; LDL-C, low density lipoprotein cholesterol. 
Table 2: The prevalence of carotid plaque by Demographical Characteristics and risk factors for all participants in this study.

\begin{tabular}{|c|c|c|c|}
\hline Characteristics/Risk factors & n (\%) & Statistic & $\boldsymbol{P}$ \\
\hline Gender: & $1574(41.5)$ & 80.516 & $<0.001$ \\
\hline Men & $782(50.1)$ & & \\
\hline Women & $792(35.5)$ & & \\
\hline Age group: & & 335.127 & $<0.001$ \\
\hline $45 \sim 54$ years & $281(22.7)$ & & \\
\hline $55 \sim 64$ years & $684(45.2)$ & & \\
\hline $65 \sim 74$ years & $390(53.9)$ & & \\
\hline$\geqslant 75$ years & $219(69.5)$ & & \\
\hline Education group: & & 48.071 & $<0.001$ \\
\hline 0 year & $318(48.3)$ & & \\
\hline 1 year & $759(44.8)$ & & \\
\hline$\geqslant 6$ years & $497(34.6)$ & & \\
\hline \multicolumn{4}{|l|}{ Risk factors: } \\
\hline Smoking status: & & 32.367 & $<0.001$ \\
\hline Never smoking & $1105(38.9)$ & & \\
\hline Smoking & $469(49.4)$ & & \\
\hline Alcohol consumption: & & 2.526 & 0.112 \\
\hline Never drinking & $1311(41.0)$ & & \\
\hline Drinking & $263(44.5)$ & & \\
\hline Hypertension: & & 98.154 & $<0.001$ \\
\hline Yes & $1213(47.0)$ & & \\
\hline No & $361(29.9)$ & & \\
\hline Diabetes: & & 33.486 & $<0.001$ \\
\hline Yes & $282(52.9)$ & & \\
\hline No & $1263(39.6)$ & & \\
\hline BMI: & & 11.964 & 0.008 \\
\hline Underweight & $38(57.6)$ & & \\
\hline Normal & $539(43.8)$ & & \\
\hline Overweight & $646(40.3)$ & & \\
\hline Obesity & $351(39.5)$ & & \\
\hline Central obesity & & 1.282 & 0.258 \\
\hline Yes & $946(60.2)$ & & \\
\hline No & $1371(62.0)$ & & \\
\hline
\end{tabular}


Table 3: The differences in measurements of risk factors between presence and absence of carotid plaque in this population.

\begin{tabular}{lccc}
\hline \multicolumn{1}{c}{ Measurement } & $\begin{array}{c}\text { Presence carotid } \\
\text { plaque }\end{array}$ & $\begin{array}{c}\text { Absence carotid } \\
\text { plaque }\end{array}$ & $\boldsymbol{P}$ \\
\hline $\mathrm{BMI}, \mathrm{Kg} / \mathrm{m}^{2}$, & $25.35(3.70)$ & $25.72(3.67)$ & 0.002 \\
$\mathrm{SBP}, \mathrm{mmHg}$ & $151.58(23.25)$ & $142.76(20.60)$ & $<0.001$ \\
$\mathrm{DBP}, \mathrm{mmHg}$ & $87.32(11.56)$ & $86.45(11.28)$ & 0.021 \\
$\mathrm{FPG}, \mathrm{mmol} / \mathrm{L}$ & $6.09(1.83)$ & $5.81(1.34)$ & $<0.001$ \\
$\mathrm{TC}, \mathrm{mmol} / \mathrm{L}$ & $4.99(1.15)$ & $4.78(1.04)$ & $<0.001$ \\
$\mathrm{TG}, \mathrm{mmol} / \mathrm{L}$ & $1.76(1.13)$ & $1.76(1.31)$ & 0.901 \\
$\mathrm{HDL}-\mathrm{C}, \mathrm{mmol} / \mathrm{L}$ & $1.45(0.45)$ & $1.46(0.47)$ & 0.582 \\
$\mathrm{LDL}-\mathrm{C}, \mathrm{mmol} / \mathrm{L}$ & $3.07(1.44)$ & $2.43(1.02)$ & $<0.001$ \\
$\mathrm{LDL}-\mathrm{C} / \mathrm{HDL}-\mathrm{C}$ & $2.33(1.54)$ & $1.79(0.87)$ & $<0.001$ \\
Waist circumference, cm & $25.35(3.70)$ & $89.11(9.11)$ & 0.199 \\
\hline
\end{tabular}

SBP, systolic blood pressure; DBP, diastolic blood pressure; BMI, body mass index; FPG, fasting plasma glucose; TC, total cholesterol; TG, triglycerides; HDL-C, high density lipoprotein cholesterol; LDL-C, low density lipoprotein cholesterol.

was higher in patients with carotid plaque for all groups except the underweight group $(P=0.509)$. However, DBP was only higher in patients with carotid plaque in the overweight group $(P=0.001)$. HDL-C was lower in patients with carotid plaque in the overweight group $(P=$ 0.022 ), while LDL-C was higher in patients with carotid plaque in all four groups (all $P<0.01$ ). Furthermore, similar to SBP, the LDL-C/HDL-C ratio was also higher in patients with carotid plaque in the normal-weight, overweight, and obese groups (all $P<0.001$ ).

\section{Multiple linear regression analysis of conventional risk factors associated with carotid plaque}

A multivariate analysis was performed to assess the relationships between carotid plaque and risk factors by BMI. Age and LDL-C were independent risk factors for carotid plaque presence in the underweight group. The risk of carotid plaque was increased by at least 10.59 -fold with older age and by 2.60-fold for each 1-unit increase in LDL-C (all $P<0.05)$. Age, SBP, FPG, and LDL-C were

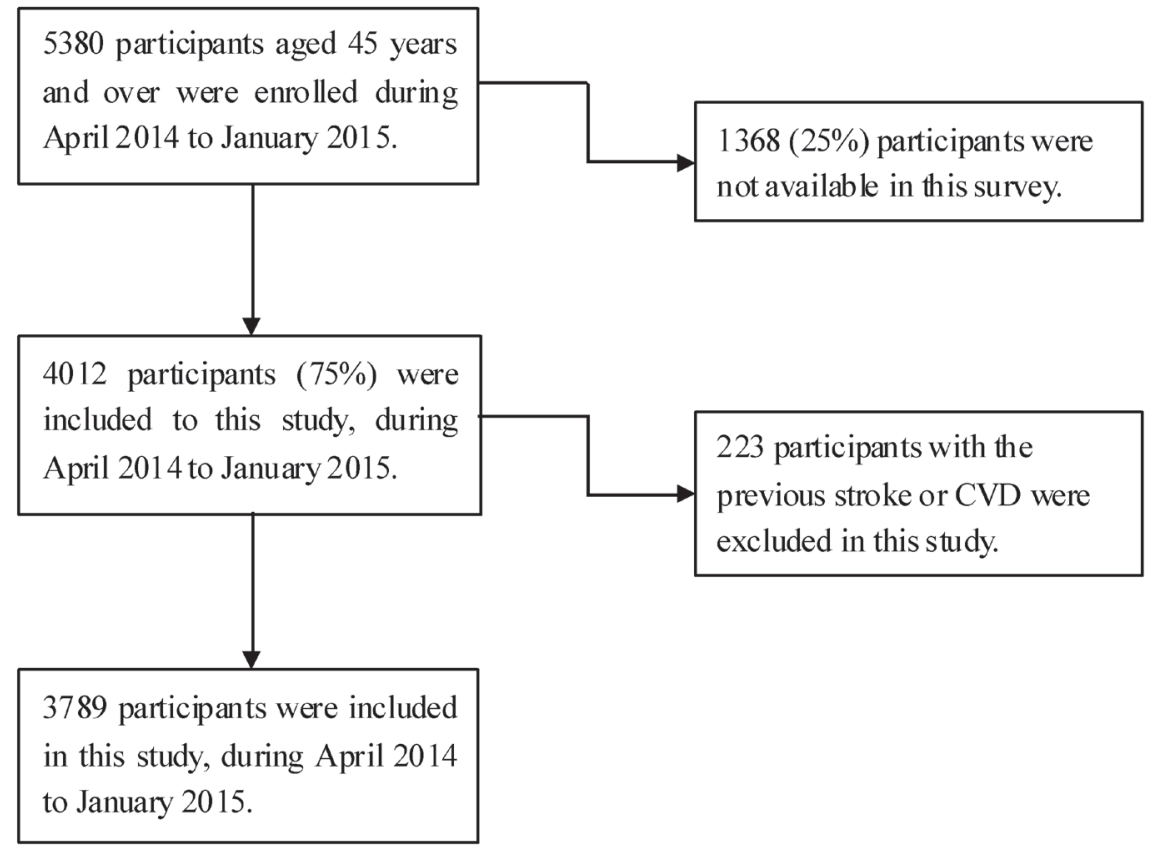

Figure 1: Flow chart of participants selection. 
Table 4: The prevalence of carotid plaque by BMI for all participants in this study.

\begin{tabular}{|c|c|c|c|c|c|c|c|c|}
\hline \multirow{2}{*}{$\begin{array}{l}\text { Characteristics/Risk } \\
\text { factors }\end{array}$} & \multicolumn{2}{|c|}{ Underweight } & \multicolumn{2}{|c|}{ Normal } & \multicolumn{2}{|c|}{ Overweight } & \multicolumn{2}{|c|}{ Obesity } \\
\hline & n $(\%)$ & $P$ & n $(\%)$ & $P$ & n (\%) & $P$ & n $(\%)$ & $P$ \\
\hline Gender: & & 0.011 & & $\begin{array}{c}< \\
0.001\end{array}$ & & $\begin{array}{c}< \\
0.001\end{array}$ & & 0.002 \\
\hline Men & $21(77.8)$ & & $280(50.3)$ & & $332(50.8)$ & & $149(46.1)$ & \\
\hline Women & $17(43.6)$ & & $259(38.4)$ & & $314(33.1)$ & & $202(35.8)$ & \\
\hline Age group: & & 0.013 & & $\begin{array}{c}< \\
0.001\end{array}$ & & $\begin{array}{c}< \\
0.001\end{array}$ & & $\begin{array}{c}< \\
0.001\end{array}$ \\
\hline $45 \sim 54$ years & $2(18.2)$ & & $81(22.6)$ & & $112(21.3)$ & & $86(25.4)$ & \\
\hline $55 \sim 64$ years & $14(60.9)$ & & $218(45.8)$ & & $283(44.0)$ & & $169(45.4)$ & \\
\hline $65 \sim 74$ years & $15(78.9)$ & & $140(55.3)$ & & $163(51.9)$ & & $52.2(72)$ & \\
\hline$\geqslant 75$ years & $7(53.8)$ & & $100(69.4)$ & & $88(73.9)$ & & $24(61.5)$ & \\
\hline Education group: & & 0.090 & & $\begin{array}{c}< \\
\cap \cap \cap 1\end{array}$ & & 0.001 & & 0.008 \\
\hline 0 year & $15(62.5)$ & & $118(52.4)$ & & $123(46.6)$ & & $62(42.5)$ & \\
\hline 1 year & $17(68.0)$ & & $267(46.9)$ & & $300(42.7)$ & & $175(44.0)$ & \\
\hline$\geqslant 6$ years & $6(35.3)$ & & $154(35.2)$ & & $223(35.0)$ & & $114(33.1)$ & \\
\hline \multicolumn{9}{|l|}{ Risk factors: } \\
\hline Hypertension & & 0.805 & & $\begin{array}{c}< \\
0.001\end{array}$ & & $\begin{array}{c}< \\
0.001\end{array}$ & & $\begin{array}{c}< \\
0.001\end{array}$ \\
\hline Yes & $18(60.0)$ & & $373(50.3)$ & & $512(46.3)$ & & $310(43.8)$ & \\
\hline No & $20(55.6)$ & & $166(33.8)$ & & $134(26.9)$ & & $41(22.7)$ & \\
\hline Diabetes & & & & $\begin{array}{c}< \\
0.001\end{array}$ & & $\begin{array}{c}< \\
0.001\end{array}$ & & 0.020 \\
\hline Yes & - & & $68(61.3)$ & & $132(53.7)$ & & $82(46.6)$ & \\
\hline No & $38(58.5)$ & & $462(42.0)$ & & $498(37.6)$ & & $265(37.7)$ & \\
\hline Current smoking & & 0.090 & & 0.001 & & $\begin{array}{c}< \\
0.001\end{array}$ & & 0.044 \\
\hline Yes & $12(75.0)$ & & $173(50.7)$ & & $199(49.3)$ & & $85(45.2)$ & \\
\hline No & $26(52.0)$ & & $366(41.1)$ & & $447(37.3)$ & & $266(38.0)$ & \\
\hline Alcohol drinking & & 0.451 & & 0.646 & & 0.410 & & 0.179 \\
\hline Yes & $6(75.0)$ & & $94(45.2)$ & & $112(42.6)$ & & $51(45.5)$ & \\
\hline No & $32(55.2)$ & & $445(43.5)$ & & $534(39.9)$ & & $300(38.7)$ & \\
\hline Central obesity & & 0.397 & & 0.055 & & 0.864 & & 0.169 \\
\hline Yes & - & & $137(48.9)$ & & $469(40.2)$ & & $340(39.2)$ & \\
\hline No & $35(61.4)$ & & $402(42.2)$ & & $177(40.7)$ & & $11(55.0)$ & \\
\hline
\end{tabular}


Table 5: The mean value of measurements in groups with or without carotid plaque by BMI group.

\begin{tabular}{|c|c|c|c|c|c|c|c|c|}
\hline \multirow{2}{*}{ Risk factors } & \multicolumn{2}{|c|}{ Underweight } & \multicolumn{2}{|c|}{ Normal } & \multicolumn{2}{|c|}{ Overweight } & \multicolumn{2}{|c|}{ Obesity } \\
\hline & $\begin{array}{l}\text { Means } \\
\text { (SD) }\end{array}$ & $\mathbf{P}$ & $\begin{array}{l}\text { Means } \\
\text { (SD) }\end{array}$ & $\boldsymbol{P}$ & $\begin{array}{l}\text { Means } \\
\text { (SD) }\end{array}$ & $P$ & $\begin{array}{l}\text { Means } \\
\text { (SD) }\end{array}$ & $\boldsymbol{P}$ \\
\hline Waist circumference, $\mathrm{cm}$ & & 0.608 & & $<0.001$ & & 0.001 & & 0.169 \\
\hline $\mathrm{CP}$ & 73.800 & & 82.627 & & 90.683 & & 99.433 & \\
\hline No-CP & 72.740 & & 81.300 & & 89.735 & & 98.790 & \\
\hline $\mathrm{SBP}, \mathrm{mmHg}$ & & 0.509 & & $<0.001$ & & $<0.001$ & & $<0.001$ \\
\hline $\mathrm{CP}$ & 138.293 & & 149.229 & & 152.650 & & 154.669 & \\
\hline No-CP & 134.101 & & 139.174 & & 142.652 & & 148.018 & \\
\hline DBP, mmHg & & 0.959 & & 0.139 & & 0.001 & & 0.885 \\
\hline $\mathrm{CP}$ & 78.272 & & 84.400 & & 88.424 & & 90.739 & \\
\hline No-CP & 78.435 & & 83.484 & & 86.481 & & 90.628 & \\
\hline $\mathrm{TC}, \mathrm{mmol} / \mathrm{L}$ & & 0.143 & & $<0.001$ & & 0.003 & & 0.125 \\
\hline $\mathrm{CP}$ & 4.923 & & 4.972 & & 4.962 & & 5.075 & \\
\hline No-CP & 4.636 & & 4.614 & & 4.802 & & 4.953 & \\
\hline $\mathrm{TG}, \mathrm{mmol} / \mathrm{L}$ & & 0.971 & & 0.241 & & 0.355 & & 0.312 \\
\hline $\mathrm{CP}$ & 1.192 & & 1.439 & & 1.870 & & 2.099 & \\
\hline No-CP & 1.196 & & 1.380 & & 1.810 & & 2.195 & \\
\hline $\mathrm{HDL}-\mathrm{C}, \mathrm{mmol} / \mathrm{L}$ & & 0.878 & & 0.801 & & 0.022 & & 0.575 \\
\hline $\mathrm{CP}$ & 1.840 & & 1.588 & & 1.379 & & 1.331 & \\
\hline No-CP & 1.859 & & 1.594 & & 1.433 & & 1.314 & \\
\hline LDL-C, $\mathrm{mmol} / \mathrm{L}$ & & 0.002 & & $<0.001$ & & $<0.001$ & & $<0.001$ \\
\hline $\mathrm{CP}$ & 2.910 & & 3.041 & & 3.070 & & 3.136 & \\
\hline No-CP & 1.883 & & 2.285 & & 2.473 & & 2.571 & \\
\hline $\mathrm{FPG}, \mathrm{mmol} / \mathrm{L}$ & & 0.744 & & $<0.001$ & & $<0.001$ & & 0.100 \\
\hline $\mathrm{CP}$ & 5.303 & & 5.887 & & 6.179 & & 6.308 & \\
\hline No-CP & 5.258 & & 5.503 & & 5.878 & & 6.112 & \\
\hline LDL-C/HDL-C ratio & & 0.057 & & $<0.001$ & & $<0.001$ & & $<0.001$ \\
\hline $\mathrm{CP}$ & 1.661 & & 2.129 & & 2.443 & & 2.511 & \\
\hline No-CP & 1.191 & & 1.539 & & 1.842 & & 2.059 & \\
\hline
\end{tabular}

SBP, systolic blood pressure; DBP, diastolic blood pressure; FPG, fasting plasma glucose; TC, total cholesterol; TG, triglycerides; HDL-C, high density lipoprotein cholesterol; LDL-C, low density lipoprotein cholesterol

positively associated with carotid plaque in the normalweight group. The risk of carotid plaque increased by at least 1.18 -fold with older age and by $61 \%$ for each 1 -unit increase in LDL-C (all $P<0.001$ ). SBP and FPG showed a slight association with carotid plaque (OR, 1.01 and 1.11;
$P=0.005$ and 0.046 , respectively). Male sex, older age, SBP, and LDL-C were statistically significantly correlated with carotid plaque presence in the overweight and obese groups (all $P<0.05$ ). SBP also showed a weak association with carotid plaque in the overweight and obese groups 
Table 6: Adjusted OR (95\% CI) of risk factors association with carotid plaque by BMI group.

\begin{tabular}{|c|c|c|c|c|c|c|c|c|c|}
\hline \multirow{2}{*}{ Risk factors } & \multirow{2}{*}{ References } & \multicolumn{2}{|l|}{ Underweight } & \multicolumn{2}{|l|}{ Normal } & \multicolumn{2}{|l|}{ Overweight } & \multicolumn{2}{|l|}{ Obesity } \\
\hline & & OR (95\% CI) & $P$ & OR $(95 \%$ CI) & $P$ & OR $(95 \%$ CI) & $P$ & OR (95\% CI) & $P$ \\
\hline Men & Women & $2.68(0.75,9.56)$ & 0.130 & $1.37(0.99,1.91)$ & 0.058 & $1.97(1.45,2.68)$ & $<0.001$ & $1.77(1.18,2.66)$ & 0.006 \\
\hline Age group & $45 \sim 54$ years & & & & & & & & \\
\hline $55 \sim 64$ years & & $12.73(1.49,109.09)$ & 0.020 & $2.18(1.53,3.10)$ & $<0.001$ & $2.67(1.98,3.60)$ & $<0.001$ & $2.18(1.53,3.10)$ & $<0.001$ \\
\hline $65 \sim 74$ years & & $18.99(1.99,181.13)$ & 0.011 & $2.95(1.92,4.54)$ & $<0.001$ & $3.17(2.18,4.63)$ & $<0.001$ & $2.34(1.45,3.76)$ & $<0.001$ \\
\hline$\geq 75$ years & & $11.59(1.17,115.00)$ & 0.036 & $5.42(3.12,9.41)$ & $<0.001$ & $7.24(4.13,12.68)$ & $<0.001$ & $4.35(2.01,9.43)$ & $<0.001$ \\
\hline Education group & $\geq 6$ years & & & & & & & & \\
\hline 0 years & & - & - & $0.90(0.58,1.39)$ & 0.630 & $0.90(0.62,1.31)$ & 0.593 & $1.16(0.71,1.87)$ & 0.561 \\
\hline $1 \sim 6$ years & & - & - & $1.01(0.74,1.39)$ & 0.944 & $0.98(0.75,1.28)$ & 0.874 & $1.21(0.85,1.72)$ & 0.282 \\
\hline Smoking & $\begin{array}{l}\text { Never } \\
\text { smoking }\end{array}$ & - & - & $1.37(0.97,1.94)$ & 0.076 & $1.20(0.86,1.67)$ & 0.274 & $0.93(0.60,1.45)$ & 0.753 \\
\hline WC & - & - & - & $1.01(0.99,1.03)$ & 0.336 & $0.99(0.97,1.01))$ & 0.447 & - & - \\
\hline SBP & - & - & - & $1.01(1.00,1.02)$ & 0.005 & $1.02(1.01,1.03)$ & $<0.001$ & $1.01(1.00,1.02)$ & 0.004 \\
\hline DBP & & - & - & - & - & $0.99(0.98,1.00)$ & 0.156 & - & - \\
\hline FPG & - & - & - & $1.11(1.00,1.23)$ & 0.046 & $1.06(0.99,1.14)$ & 0.092 & - & - \\
\hline HDL-C & & - & - & - & - & $0.81(0.61,1.07)$ & 0.141 & - & - \\
\hline LDL-C & - & $3.60(1.34,9.72)$ & 0.011 & $1.61(1.32,1.97)$ & $<0.001$ & $1.61(1.45,1.79)$ & $<0.001$ & $1.41(1.14,1.74)$ & 0.001 \\
\hline LDL-C/HDL-C & - & - & - & $1.10(0.87,1.40)$ & 0.414 & - & - & $1.07(0.84,1.36)$ & 0.612 \\
\hline
\end{tabular}

SBP, systolic blood pressure; DBP, diastolic blood pressure; FPG, fasting plasma glucose; HDL-C, high density lipoprotein cholesterol; LDL-C, low density lipoprotein cholesterol

(OR, 1.02 and 1.01, respectively). Moreover, the significant relationship between education level, smoking, waist circumference, DBP, and the LDL-C/HDL-C ratio disappeared following adjustment for other confounding factors (all $P>0.05$ ) (Table 6).

\section{DISCUSSION}

This is the first report to describe the associations between BMI and carotid plaque in a low-income population with a high incidence of stroke in rural China. Significant correlations were observed between the presence of carotid plaque and male sex, older age, SBP, FPG, and LDL-C among the four BMI subgroups. Of those factors, a positive association was found between carotid plaque and both older age and LDL-C among all four subgroups. Male sex increased the risk of carotid plaque in the overweight and obese groups. SBP was an independent risk factor for carotid plaque in the normalweight, overweight, and obese groups. Moreover, FPG was only significantly positively correlated with carotid plaque in the normal-weight group. No significant links between carotid plaque and education level, cigarette smoking, waist circumference, DBP, HDL-C, or the LDL-C/HDL-C ratio were observed in this study.

Several studies demonstrated that age is a strong risk factor for carotid plaque $[6,9,10,22]$. In a traditional vascular risk factor model, Kuo et al. [6] demonstrated that older age was significantly associated with carotid plaque presence $(\mathrm{B}=0.332, P<0.0001)$. A communitybased study from Iceland [9] explored the prevalence and determinants of carotid plaque in 6524 participants aged 25-69 years. A multinomial logistic regression analysis in that study showed that older age was a risk factor for carotid plaque. The risk of carotid plaque increased by 0.97 -fold for each 5 -year increase in age (95\% CI, 1.83 $2.12, P<0.001)$. Our study also showed that older age was a strong risk factor for CP. Older age was significantly correlated with carotid plaque among all four BMI subgroups. The risk of carotid plaque presence increased by at least 1.18 -fold for older individuals compared to that in younger individuals.

The relationship between serum lipid profiles and carotid plaque has been widely studied worldwide. However, there are conflicting results with respect to the 
relationship between LDL-C and carotid plaque. Mi et al. [23] reported that LDL-C had no significant association with CP. They recruited 22,222 urban or rural residents who were at high risk of stroke and not using any drugs that might affect serum lipids. After controlling for age, sex, education, traditional risk factors, history of stroke, and family history of stroke, a multivariate analysis showed no association between carotid plaque and LDL-C (OR, 0.976; 95\% CI, 0.914-1.043; $P=0.475$ ). In contrast to those results, Yang et al. [19] concluded that LDL-C was a risk factor for carotid plaque presence (OR, $1.325 ; 95 \% \mathrm{CI}, 1.046-1.821 ; P=0.033$ ) in a multivariate model in which the presence of carotid plaque was the dependent variable and age, LDL-C, and HDL-C were the independent variables. The results of the present study showed that LDL-C was a strong risk factor for carotid plaque among the four weight groups. The risk of carotid plaque developing increased by $\geq 41 \%$ for each $1-\mathrm{mmol} / \mathrm{L}$ increase in the serum concentration of LDL-C. The underlying mechanism might be explained by oxidized LDL-C, which could enter and accumulate within the arterial walls and be involved in the inflammatory process in atherosclerosis [24].

Several studies have shown that male sex is a risk factor for carotid plaque [6,9]. In a traditional vascular risk factor model, male sex contributed to carotid plaque development (B $=0.084, P<0.002)$ [6]. Sturlaugsdottir et al. [9] reported that male sex was a risk factor for CP (OR, 1.53; 95\% CI, 1.22-1.92, $P<0.001$ ). The results of the present study were consistent with those results. Male sex was a risk factor for carotid plaque among both the overweight and obese groups. However, the significant relationship between male sex and carotid plaque among the underweight and normal-weight groups disappeared in the multivariate model, which may be explained by the observation that the numbers of carotid plaque patients in the underweight and normal-weight groups were too small to reach statistical significance.

Gardener et al. [6] and Sturlaugsdottir et al. [9] also showed that SBP significantly affected the development of carotid plaque. In the study by Gardener et al., SBP positively increased the risk of carotid plaque $(\mathrm{B}=0.159$, $P<0.0001)$. Sturlaugsdottir et al. revealed that each 10$\mathrm{mmHg}$ increase in SBP increased the risk of carotid plaque by $20 \%(95 \%$ CI, $1.15-1.26 ; P<0.001)$. Similar to their research, our study also showed that SBP was a risk factor for carotid plaque in the normal-weight, overweight, and obese groups. However, the effect of SBP on carotid plaque development was limited; the risk of carotid plaque increased by $\leq 2 \%$ for each 1-unit increase in SBP. Furthermore, Yin et al. [25] conducted a populationbased study of 4,992 individuals in Hang Zhou, China, and they reported that FPG was a risk factor for carotid plaque in both men and women (OR, 1.49; 95\% CI, 1.21$1.84, P<0.0001$ and $\mathrm{OR}, 1.93 ; 95 \% \mathrm{CI}, 1.31-2.84, P=$ 0.001 , respectively). Consistent with Yin et al.'s report, the present study showed that FPG was a risk factor for carotid plaque in the normal-weight group, although FPG only slightly increased the risk for carotid plaque (by $11 \%)$.

In addition, although previous studies have shown that education level [6], cigarette smoking $[6,9,10,12$ 14], DBP [26, 27], HDL-C [10, 19], and the LDL-C/ HDL-C ratio $[10,19]$ were predictors of carotid plaque, no significant link between carotid plaque and education level, cigarette smoking, DBP, HDL-C, or the LDL-C/ HDL-C ratio was observed in this study. This finding might be explained by the fact that the previous study did not analyze the association between those variables with carotid plaque after stratification by BMI, which could have led to the disappearance of statistical significance.

This study has some limitations. (1) An inherent limitation of our cross-sectional study design is that it cannot determine the causal correlation between carotid plaque and the variables found to be significant; thus, further longitudinal studies are warranted to determine causality. (2) The study population consisted of lowincome adults $\geq 45$ years living in a rural area of northern China, so the conclusions of the present study may not be generalizable to other populations. However, considering that low-income, rural residents comprise $>50 \%$ of the total population of China, the results of the present study are important for further study. (3) We did not collect information regarding medication use for all subjects; however, due to the participants' low socioeconomic status, the frequency of medication use was lower in this population, so it is likely that it did not affect the validity of the present results.

This is the first population-based study to explore the association between BMI and carotid plaque presence in rural China. All participants were low-income residents with low educational attainment aged 45 years and older. To our knowledge, no similar report has revealed an association between BMI and carotid plaque. The multivariate analysis showed that older age and LDL-C were risk factors for carotid plaque among all four BMI subgroups. Male sex was a risk factor for carotid plaque presence in the overweight and obese groups. SBP was an independent risk factor for carotid plaque in the normalweight, overweight, and obese groups. FPG was a risk factor for carotid plaque in the normal-weight group.

LDL-C was a strong independent risk factor for carotid plaque among all groups. Therefore, the findings of this study suggest that it is necessary to control LDL-C in this population. In addition, controlling SBP and FPG will also assist in reducing the carotid plaque risk. Furthermore, our study provided specific information for clinicians regarding specific preventive strategies for different populations by BMI. 


\section{MATERIALS AND METHODS}

\section{Study population}

The present study was a population-based, crosssectional study conducted from April 2014 to January 2015. The enrolled population was from the Tianjin Brain Study [28-31]. In brief, the total population comprised 14,251 participants from among 18 administrative villages in rural Tianjin, China. About $95 \%$ of participants were low-income farmers, with an average disposable annual income $<\$ 1600$ US in 2014 [32]. Residents aged $\geq 45$ years without CVDs were included in this study, while those with a previous history of CVD were excluded.

All investigative protocols were approved by the ethics committee of Tianjin Medical University General Hospital; the methods were carried out in accordance with the approved guidelines, and informed consent was obtained from all participants.

\section{Information collection and risk factor definitions}

All variables in this study were obtained by trained epidemiological researchers through face-to-face interviews. A pre-specified questionnaire was used to collect all information for this study.

Demographic information, including name, sex, date of birth, and educational level, were derived from previous records. All participants were separated into four age groups: 45-54 years, 55-64 years, 65-74 years, and $\geq 75$ years. Educational level was classified into three groups according to the length of the individuals' formal education: illiteracy (without a formal education), 1-6 years group, and $>6$ years group.

Previous individual and family medical histories, including of hypertension, DM, stroke, transient ischemic attack, and coronary heart disease, were obtained according to patient self-report or previous records.

Lifestyle characteristics of interest included cigarette smoking and alcohol consumption. Cigarette smoking was defined as smoking more than 1 cigarette per day for at least 1 year, and participants were categorized as never smokers and smokers. Alcohol consumption was defined as drinking more than 500 grams of alcohol per week for at least 1 year, and participants were divided into the never alcohol consumption group and alcohol consumption group.

\section{Physical examination}

Measurements of blood pressure (including SBP and DBP), height, and weight were measured in the local village clinic during the baseline survey; levels of
FPG, TC, TG, HDL-C, and LDL-C in the serum were assessed at the Ji County People's Hospital. Carotid ultrasonography and 12-lead echocardiography were also conducted by a professional. BMI was calculated as the participant's weight $(\mathrm{kg})$ divided by the square of height $\left(\mathrm{m}^{2}\right)$.

Hypertension was defined as SBP $\geq 140 \mathrm{mmHg}$, $\mathrm{DBP} \geq 90 \mathrm{mmHg}$, or taking medication for hypertension. DM was defined as FPG $\geq 7.0 \mathrm{mmol} / \mathrm{L}$ or taking insulin or oral hypoglycemic medications.

\section{Ultrasonography measurements}

One trained technician blinded to individuals' previous disease histories performed all ultrasound examinations. The patients were examined when they were in the supine position using B-mode ultrasonography (Terason 3000; Burlington, MA, US) with a 5-12-MHz linear array transducer. Plaques are focal structures that encroach into the arterial lumen by at least $0.5 \mathrm{~mm}$ or $50 \%$ of the surrounding intima-media thickness, or demonstrate a thickness of $>1.5 \mathrm{~mm}$, as measured from the intimalumen interface to the media-adventitia interface [33]. Subjects with carotid plaque were defined as having at least one lesion, not based on the total number of carotid plaques.

\section{Statistical analyses}

Continuous variables were presented as means with standard deviations, and Student's $t$-test or an analysis of variance was used to compare differences between two groups or multiple groups, respectively. Categorical variables were presented as numbers with frequencies and were compared using the chi-squared test. Multiple linear regression analyses were used to evaluate the associations between traditional risk factors and the presence of carotid plaques. We performed multivariate logistic regression analyses to evaluate the determinants of carotid plaque after adjusting for other confounding factors. The results are presented as adjusted ORs and 95\% CIs. $P$ values $<0.05$ in two-tailed tests were considered statistically significant. SPSS for Windows (version 19.0; SPSS Inc., Chicago, IL, USA) was used for analyses.

\section{Abbreviations}

BMI, body mass index; CI, confidence interval; CVD, cardiovascular disease; DBP, diastolic blood pressure; DM, diabetes mellitus; FPG, fasting plasma glucose; HDL-C, high-density lipoprotein cholesterol; LDL-C, low-density lipoprotein cholesterol; OR, odds ratio; TC, total cholesterol; TG, triglycerides; SBP, systolic blood pressure. 


\section{Author contributions}

$\mathrm{JW}, \mathrm{XN}$, and $\mathrm{BL}$ contributed to the study design. JW and XN performed data collection, data interpretation, and data analysis. $\mathrm{BL}$ and $\mathrm{XN}$ performed critical review. YL contributed to drafting of the article. YL, LS, ZM, MS, HG, JN, YW, JT, and JW performed data collection, case diagnoses, and confirmation of case diagnoses.

\section{ACKNOWLEDGMENTS}

The authors would like to thank all participants and local health workers.

\section{CONFLICTS OF INTEREST}

The authors declare that they have no conflicts of interest.

\section{REFERENCES}

1. WHO. Cardiovascular diseases (CVDs). 2016.

2. National Center for Cardiovascular Diseases, China. Report on Cardiovascular Diseases in China, Encyclopedia of China Publishing House; 2015.

3. Feigin VL, Forouzanfar $\mathrm{MH}$, Krishnamurthi R, Mensah GA, Connor M, Bennett DA, Moran AE, Sacco RL, Anderson L, Truelsen T, O'Donnell M, Venketasubramanian N, Barker-Collo S, et al. Global and regional burden of stroke during 1990-2010: findings from the Global Burden of Disease Study 2010. Lancet. 2014; 383: 245-254.

4. Oh PC, Han SH, Kim JS, Park JB, Koh KK. Carotid plaque in absence of traditional and non-traditional cardiovascular risk factors. Int J Cardiol. 2010; 143: e57-59.

5. Xie W, Wu Y, Wang W, Zhao D, Liang L, Wang M, Yang Y, Sun J, Shi P, Huo Y. A longitudinal study of carotid plaque and risk of ischemic cardiovascular disease in the Chinese population. J Am Soc Echocardiogr. 2011; 24: 729737.

6. Kuo F, Gardener H, Dong C, Cabral D, Della-Morte D, Blanton SH, Elkind MS, Sacco RL, Rundek T. Traditional cardiovascular risk factors explain the minority of the variability in carotid plaque. Stroke. 2012; 43: 17551760.

7. Naqvi TZ, Lee MS. Carotid intima-media thickness and plaque in cardiovascular risk assessment. JACC Cardiovasc Imaging. 2014; 7: 1025-1038.

8. Ghouri N, Purves D, Deans KA, Logan G, McConnachie A, Wilson J, Gill JM, Sattar N. An investigation of twodimensional ultrasound carotid plaque presence and intima media thickness in middle-aged South Asian and European men living in the United kingdom. PLoS One. 2015; 10: e0123317.
9. Sturlaugsdottir R, Aspelund T, Bjornsdottir G, Sigurdsson S, Thorsson B, Eiriksdottir G, Gudnason V. Prevalence and determinants of carotid plaque in the cross-sectional REFINE-Reykjavik study. BMJ Open. 2016;6: e012457.

10. Prati P, Vanuzzo D, Casaroli M, Di Chiara A, De Biasi F, Feruglio GA, Touboul PJ. Prevalence and determinants of carotid atherosclerosis in a general population. Stroke. 1992;23: 1705-1711.

11. Gardener H, Della Morte D, Elkind MS, Sacco RL, Rundek T. Lipids and carotid plaque in the Northern Manhattan Study (NOMAS). BMC Cardiovasc Disord. 2009; 9: 55.

12. Tattersall MC, Gassett A, Korcarz CE, Gepner AD, Kaufman JD, Liu KJ, Astor BC, Sheppard L, Kronmal RA, Stein JH. Predictors of carotid thickness and plaque progression during a decade: the Multi-Ethnic Study of Atherosclerosis. Stroke. 2014; 45: 3257-3262.

13. Högberg $D$, Kragsterman $B$, Björck $M$, Tjärnström J, Wanhainen A. Carotid artery atherosclerosis among 65-year-old Swedish men - a population-based screening study. Eur J Vasc Endovasc Surg. 2014; 48: 5-10.

14. Yang D, Iyer S, Gardener H, Della-Morte D, Crisby M, Dong C, Cheung K, Mora-McLaughlin C, Wright CB, Elkind MS, Sacco RL, Rundek T. Cigarette Smoking and Carotid Plaque Echodensity in the Northern Manhattan Study. Cerebrovasc Dis. 2015;40: 136-143.

15. West HW, Juonala M, Gall SL, Kähönen M, Laitinen T, Taittonen L, Viikari JS, Raitakari OT, Magnussen CG. Exposure to parental smoking in childhood is associated with increased risk of carotid atherosclerotic plaque in adulthood: the Cardiovascular Risk in Young Finns Study. Circulation. 2015;131: 1239-1246.

16. Lim GB. Risk factors: Carotid plaque in adulthood after childhood exposure to parental smoking. Nat Rev Cardiol. 2015; $12: 319$.

17. Li Q, Zhou Y, Dong K, Wang A, Yang X, Zhang C, Zhu $\mathrm{Y}$, Wu S, Zhao X. The Association between Serum Uric Acid Levels and the Prevalence of Vulnerable Atherosclerotic Carotid Plaque: A Cross-sectional Study. Sci Rep. 2015;5:10003.

18. Zhou Y, Wang D, Yang X, Wang A, Gao X, Guo Y, Wu $\mathrm{S}$, Zhao X. Effect of menopausal status on carotid intimamedia thickness and presence of carotid plaque in Chinese women generation population. Sci Rep. 2015;5: 8076.

19. Yang C, Sun Z, Li Y, Ai J, Sun Q, Tian Y. The correlation between serum lipid profile with carotid intima-media thickness and plaque. BMC Cardiovasc Disord. 2014;14: 181.

20. Zhang XT, Zhao D, Liu J, Feng LQ, Sun JY, Wang M, Liu J, Wang W. [Association between both triglyceride level, newly identified carotid plaque and middle-aged and elderly population, Beijing]. [Article in Chinese]. Zhonghua Liu Xing Bing Xue Za Zhi. 2012;33: 455-459.

21. Lee EJ, Kim HJ, Bae JM, Kim JC, Han HJ, Park CS, Park NH, Kim MS, Ryu JA. Relevance of common carotid 
intima-media thickness and carotid plaque as risk factors for ischemic stroke in patients with type 2 diabetes mellitus. AJNR Am J Neuroradiol. 2007; 28: 916-919.

22. Lanktree MB, Hegele RA, Schork NJ, Spence JD. Extremes of unexplained variation as a phenotype: an efficient approach for genome-wide association studies of cardiovascular disease. Circ Cardiovasc Genet. 2010; 3: 215-221.

23. Mi T, Sun S, Zhang G, Carora Y, Du Y, Guo S, Cao M, Zhu Q, Wang Y, Sun Q, Wang X, Qu C. Relationship between dyslipidemia and carotid plaques in a high-strokerisk population in Shandong Province, China. Brain Behav. 2016;6: e00473.

24. Elkind MS, Cheng J, Boden-Albala B, Paik MC, Sacco RL; Northern Manhattan Stroke Study. Elevated white blood cell count and carotid plaque thickness : the northern manhattan stroke study. Stroke. 2001; 32: 842-849.

25. Yin JH, Song ZY, Shan PF, Xu J, Ye ZM, Xu XH, Zhang SZ, Liang Q, Zhao Y, Ren Z, Yu YP. Age- and genderspecific prevalence of carotid atherosclerosis and its association with metabolic syndrome in Hangzhou, China. Clin Endocrinol (Oxf). 2012; 76: 802-809.

26. Johnsen SH, Mathiesen EB, Joakimsen O, Stensland E, Wilsgaard T, Løchen ML, Njølstad I, Arnesen E. Carotid atherosclerosis is a stronger predictor of myocardial infarction in women than in men: a 6-year follow-up study of 6226 persons: the Tromsø Study. Stroke. 2007; 38: $2873-$ 2880.

27. Murias Quintana E, Vega Valdés P, Morales Deza E, Gil Garcia A, Cuellar H, Costilla García S, Cadenas Rodríguez M, Delgado MG, Peña Suárez J, Santamarta Liébana E, López-García A. Analysis of endovascular treatment of ruptured aneurysms of the middle cerebral artery compared to other anatomical locations. Interv Neuroradiol. 2016;22: 649-653.
28. Wang J, Ning X, Yang L, Lu H, Tu J, Jin W, Zhang $\mathrm{W}, \mathrm{Su}$ TC. Trends of hypertension prevalence, awareness, treatment and control in rural areas of northern China during 1991-2011. J Hum Hypertens. 2014; 28: 25-31.

29. Wang J, Ning X, Yang L, Tu J, Gu H, Zhan C, Zhang W, Su TC. Sex differences in trends of incidence and mortality of first-ever stroke in rural Tianjin, China, from 1992 to 2012. Stroke. 2014; 45: 1626-1631.

30. Wang J, An Z, Li B, Yang L, Tu J, Gu H, Zhan C, Liu B, Su $\mathrm{TC}$, Ning $\mathrm{X}$. Increasing stroke incidence and prevalence of risk factors in a low-income Chinese population. Neurology. 2015; 84: 374-381.

31. Ning $X$, Zhan C, Yang Y, Yang L, Tu J, Gu H, Su TC, Wang J. Secular trends in prevalence of overweight and obesity among adults in rural Tianjin, China from 1991 to 2011: a population-based study. PLoS One. 2014; 9: e116019.

32. National Health and Family Planning Commission of the People's Republic of China. China Health and Family Planning Statistics Yearbook 2014, China Union Medical University Press;2015.

33. Touboul PJ, Hennerici MG, Meairs S, Adams H, Amarenco P, Bornstein N, Csiba L, Desvarieux M, Ebrahim S, Hernandez Hernandez R, Jaff M, Kownator S, Naqvi T, et al. Mannheim carotid intima-media thickness and plaque consensus (2004-2006-2011). An update on behalf of the advisory board of the $3 \mathrm{rd}$, 4th and 5 th watching the risk symposia, at the 13th, 15th and 20th European Stroke Conferences, Mannheim, Germany, 2004, Brussels, Belgium, 2006, and Hamburg, Germany, 2011. Cerebrovasc Dis. 2012; 34: 290-296. 